\title{
High-power low-divergence tapered quantum cascade lasers with plasmonic collimators
}

\section{Citation}

Blanchard, Romain, Tobias S. Mansuripur, Burc Gökden, Nanfang Yu, Mikhail Kats, Patrice Genevet, Kazuue Fujita, Tadataka Edamura, Masamichi Yamanishi, and Federico Capasso. 2013. "High-Power Low-Divergence Tapered Quantum Cascade Lasers with Plasmonic Collimators." Applied Physics Letters 102 (19): 191114. https://doi.org/10.1063/1.4806985.

\section{Permanent link}

http://nrs.harvard.edu/urn-3:HUL.InstRepos:41371387

\section{Terms of Use}

This article was downloaded from Harvard University's DASH repository, and is made available under the terms and conditions applicable to Other Posted Material, as set forth at http:// nrs.harvard.edu/urn-3:HUL.InstRepos:dash.current.terms-of-use\#LAA

\section{Share Your Story}

The Harvard community has made this article openly available.

Please share how this access benefits you. Submit a story.

Accessibility 


\title{
High-power low-divergence tapered quantum cascade lasers with plasmonic collimators
}

\author{
Romain Blanchard, ${ }^{1}$ Tobias S. Mansuripur, ${ }^{2}$ Burc Gökden, ${ }^{1}$ Nanfang Yu, ${ }^{1, a)}$ Mikhail Kats, ${ }^{1}$ \\ Patrice Genevet, ${ }^{1}$ Kazuue Fujita, ${ }^{3}$ Tadataka Edamura, ${ }^{3}$ Masamichi Yamanishi, ${ }^{3}$ \\ and Federico Capasso ${ }^{1, b)}$ \\ ${ }^{1}$ School of Engineering and Applied Sciences, Harvard University, 29 Oxford St., Cambridge, \\ Massachusetts 02138, USA \\ ${ }^{2}$ Harvard University Department of Physics, 17 Oxford St., Cambridge, Massachusetts 02138, USA \\ ${ }^{3}$ Central Research Laboratory, Hamamatsu Photonics K. K., Hamamatsu 434-8601, Japan
}

(Received 25 February 2013; accepted 2 May 2013; published online 15 May 2013)

\begin{abstract}
We demonstrate a tapered quantum cascade laser with sloped side-walls emitting a high-brightness single-lobe beam at $8.1 \mu \mathrm{m}$ with a peak power of $4 \mathrm{~W}$ at room temperature. Using a combination of high and low reflectivity facet coatings, the power output is increased to $6.2 \mathrm{~W}$ while a high beam quality is maintained. Plasmonic collimators are fabricated on the facet of the uncoated lasers without compromising power output, demonstrating the viability of this beam-shaping strategy for high-power lasers. The collimated lasers emit a beam with a more circular cross-section, which is more amenable to high-efficiency coupling into mid-infrared optical fibers. (C) 2013 AIP Publishing LLC.

[http://dx.doi.org/10.1063/1.4806985]
\end{abstract}

Quantum cascade lasers (QCLs) are infrared semiconductor light sources with an increasing number of applications, from spectroscopy ${ }^{1}$ to free-space communication ${ }^{2}$ and military countermeasures. Record performance, with Wattlevel continuous-wave output power ${ }^{3,4}$ is now regularly obtained for edge-emitting devices.

For many applications, high power output and lasing on the fundamental transverse mode are desirable. We recently showed ${ }^{5}$ that QCLs with tapered waveguides and curved wet-etched side-walls feature the high power of broad-area QCLs while maintaining a good beam quality. This improvement on earlier efforts using tapered QCLs ${ }^{6}$ was achieved by introducing higher losses for higher order lateral modes than for the fundamental mode, using a small section of straight waveguide at the narrow end of the taper and curved lossy side-walls. We reported ${ }^{5}$ a $1^{\circ}$ half-width tapered device with a peak power of $2.5 \mathrm{~W}$ at $9.5 \mu \mathrm{m}$, an $\mathrm{M}^{2}$ of 2.08 and a maximum brightness of $1.4 \mathrm{MW} \mathrm{cm} \mathrm{sr}^{-1}$. Angled cavity QCLs with broad waveguides have also been reported ${ }^{7}$ for lateral mode control, but no data was provided on the robustness of the design as driving current is increased.

In the present work, we improve these results using a different QCL material ${ }^{8}$ lasing at $8.1 \mu \mathrm{m}$ and grown by metal-organic chemical vapor deposition. A schematic topview of the waveguide design is shown in the inset of Fig. 1(a). It consists of a $0.5 \mathrm{~mm}$-long and $14 \mu \mathrm{m}$-wide narrow ridge section, followed by a $2.5 \mathrm{~mm}$-long tapered section with a $1^{\circ}$ half-angle. The waveguides were defined by wet etching two trenches approximately $20 \mu \mathrm{m}$-wide and with a depth reaching just beyond the active region $(\approx 9.5 \mu \mathrm{m})$. This resulted in sidewalls inclined at about $37^{\circ}$ with respect to the plane of the epitaxial layers. A $500 \mathrm{~nm}$-thick SiN passivation layer was then deposited by plasma-enhanced chemical

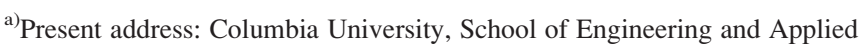
Science, NY

b)Electronic mail: capasso@seas.harvard.edu
}

vapor deposition and opened by reactive ion etching on top of the laser ridges. The contact opening is also tapered so that the width of the contact is about half the waveguide width all along the laser. Top and bottom metallization consisted of $\operatorname{Ti}(15 \mathrm{~nm})$ and $\mathrm{Au}(350 \mathrm{~nm})$ layers. Finally, an additional $4 \mu \mathrm{m}$ of gold was electroplated on the top contact to improve heat dissipation. The wafer was then cleaved into $3 \mathrm{~mm}$-long chips, which were indium-soldered epi-side up on copper heat sinks. For all the results presented here, the lasers are driven in pulsed operation, with a $10 \mathrm{kHz}$ repetition rate and $100 \mathrm{~ns}$ pulse width, and maintained at $20^{\circ} \mathrm{C}$ with a Peltier cooler.

Fig. 1(a) shows the L-I and I-V characteristics of a representative tapered QCL (laser A), as well as a Fabry-Perot (FP) QCL (i.e., non-tapered QCL, $14 \mu \mathrm{m}$-wide, $3 \mathrm{~mm}$-long) for comparison. The tapered QCL reaches $3.95 \mathrm{~W}$ peak power. For comparison, devices reported in Ref. 5 with a similar geometry and lasing at $9.5 \mu \mathrm{m}$ had a peak power of $2.5 \mathrm{~W}$. The increased performance from this earlier work is due to the different active region design and growth conditions. The slope efficiencies for the FP and the tapered QCLs are 720 and $660 \mathrm{~mW} / \mathrm{A}$, respectively. The small reduction in slope efficiency can be ascribed to higher optical losses due to the small non-adiabaticity of the taper. From the same fabrication run, we cleaved five $3 \mathrm{~mm}$-long chips, each containing four similar tapered QCLs. We measured the L-I curves of the 20 tapered lasers and found an average peak power of $4.08 \mathrm{~W}$ with a standard deviation of $0.35 \mathrm{~W}$.

The far-field profile of laser A (along the slow axis) is shown in Fig. 1(b). We observe only a small dependence on drive current across the operating range. The $\mathrm{M}^{2}$ value is calculated to be between $1.6\left(\right.$ at $\left.\mathrm{I}=2 \mathrm{I}_{\mathrm{th}}\right)$ and $1.8\left(\right.$ at $\mathrm{I}=3.5 \mathrm{I}_{\mathrm{th}}$ ) while the maximum brightness reaches $3.35 \mathrm{MW} \mathrm{cm} \mathrm{sr}^{-1}$ (details on the definitions and calculations of $\mathrm{M}^{2}$ and brightness can be found in Refs. 5, 9, and 10). Gain, temperature, or refractive index inhomogeneities in the waveguide as well as scattering from facet defects provide a coupling 

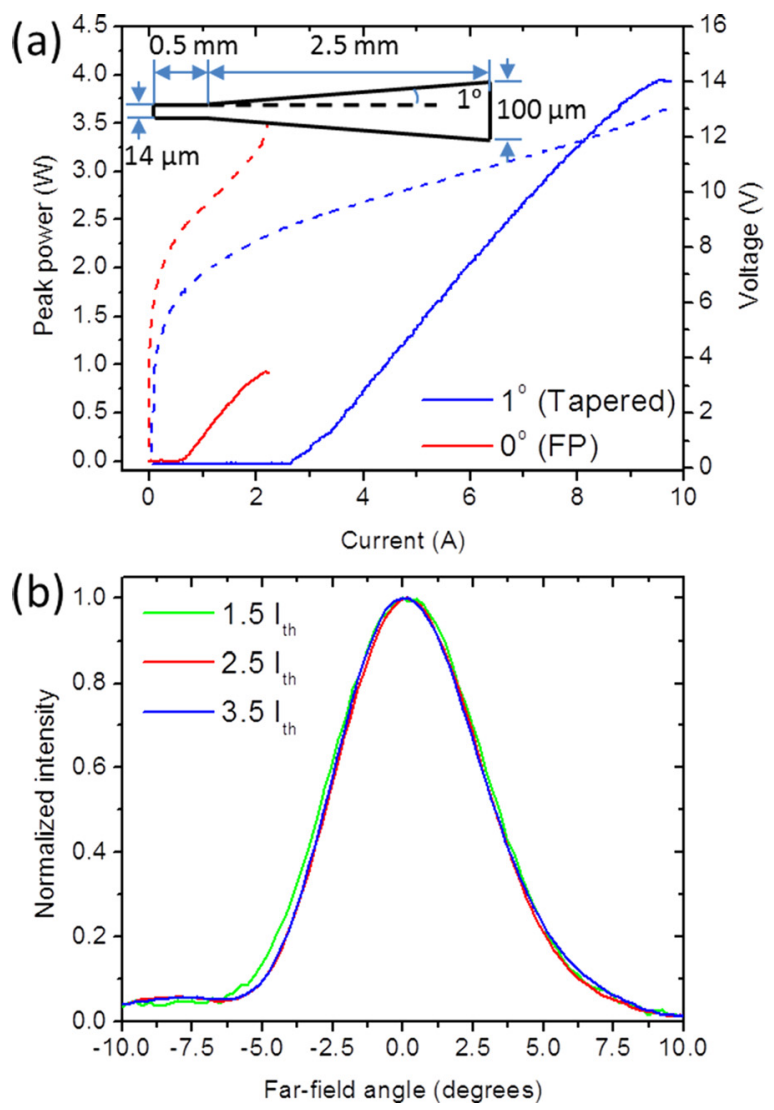

FIG. 1. (a) L-I (solid) and I-V (dashed) curves of representative FP and tapered QCLs with $1^{\circ}$ taper half-angle (laser A). The FP laser waveguide is $14 \mu \mathrm{m}$-wide and $3 \mathrm{~mm}$-long. The optical power is measured from the front (wide) facet. Inset: schematic of the waveguide structure. (b) Far-field profiles of laser A for increasing pump current. The far-fields are measured using a thermoelectrically cooled mercury-cadmium-telluride detector mounted on a rotation stage $18 \mathrm{~cm}$ away from the laser facet. The plane of the scan is parallel to the planes of the QCL epitaxial layers (slow axis).

mechanism between lateral modes and can cause the asymmetries and small side-lobes sometimes observed in the farfield. For this fabrication run, facet defects are expected to play a predominant role since the continuous thick electroplated gold contact covered the entire waveguide, which led to difficult cleaving of the wafer. This can be improved easily by leaving narrow sections of the waveguide free of electroplated gold. On some of the devices measured, the lasers could start lasing predominantly on a higher order lateral mode when driven beyond $80 \%$ to $90 \%$ of the roll-over current. This was usually also visible in the L-I curve since it translates into a change in slope efficiency. The far-field of the FP laser (not shown) was Gaussian with a full-width at half-maximum of $43.7^{\circ}$ and independent of current, from just above threshold to the roll-over current.

We then proceeded to optimize the power output of the tapered QCLs using a high reflectivity (HR) coating on the back facet and a low-reflectivity (LR) coating on the front facet. Such a strategy has already been successfully employed to optimize the output power of either long ${ }^{11}$ or short $^{12}$ cavity QCLs operating at $4.6 \mu \mathrm{m}$. The HR coating here consists of a $50 \mathrm{~nm}$-thick $\mathrm{Al}_{2} \mathrm{O}_{3}$ insulating layer followed by a $10 \mathrm{~nm}$-thick titanium adhesion layer and a $400 \mathrm{~nm}$-thick gold reflecting layer, all deposited by electronbeam evaporation. The LR coating was realized by a single layer of $\mathrm{Al}_{2} \mathrm{O}_{3}$. While $\mathrm{Al}_{2} \mathrm{O}_{3}$ starts having optical losses around $8 \mu \mathrm{m}\left(\approx 150 \mathrm{~cm}^{-1}\right)$, we chose to use this material because of its good thermal and insulating properties, its ease of deposition and its reliable adhesion to QCL facets. The refractive index ${ }^{13}$ of $\mathrm{Al}_{2} \mathrm{O}_{3}$ at $8.1 \mu \mathrm{m}$ is close to 1.37, allowing to reach reflectivities between $29 \%$ (uncoated) and 7.6\% (quarter-wave layer). We use 3.3 as the refractive index of the QCL waveguide in our calculations. Successive electron-beam evaporations resulted in an $\mathrm{Al}_{2} \mathrm{O}_{3}$ layer with increasing thickness from $740 \mathrm{~nm}$ to $1100 \mathrm{~nm}$ to $1510 \mathrm{~nm}$, corresponding, respectively, to theoretical reflectivities of $20 \%, 12 \%$, and $7.6 \%$.

The L-I characteristics of the uncoated original device (laser B) and measured after each deposition are shown in Fig. 2(a). We observe that the HR coating improves the power output by $40 \%$ to a peak power of $5.2 \mathrm{~W}$. While theoretical analyses have been employed in the literature ${ }^{11,12}$ to predict the optimal front facet reflectivity (or equivalently, the optimal laser length), they usually rely on the assumption of uniform gain saturation and photon density, which is not valid with our geometry. We measured the output power from both the front (wide) and the back (narrow) facet of uncoated tapered lasers and observed that the power emitted from the back facet is typically only about $65 \%$ of the power
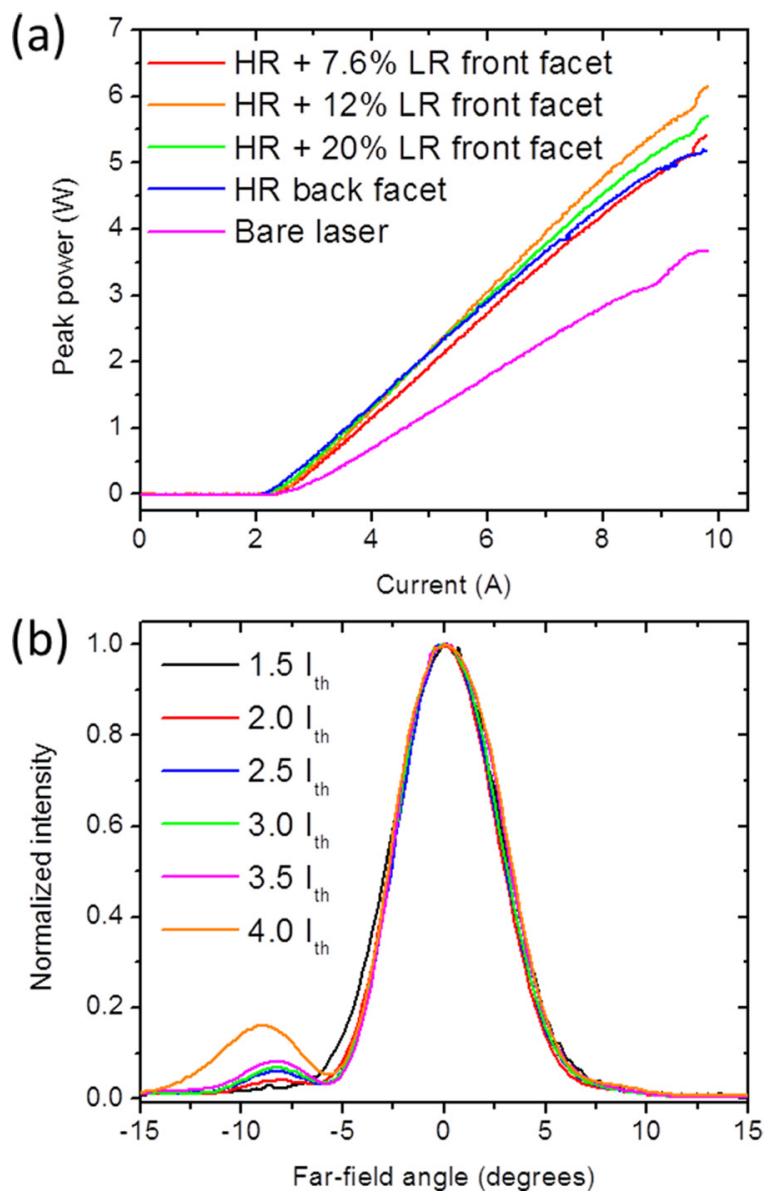

FIG. 2. (a) LI curves of a representative tapered QCL with different facet coatings. The successive thicknesses reached by the LR coating are $740 \mathrm{~nm}$, $1110 \mathrm{~nm}$, and $1510 \mathrm{~nm}$, corresponding, respectively, to theoretical reflectivities of $20 \%, 12 \%$, and $7.6 \%$. (b) Far-field profiles of the final device shown in (a), with HR coating on the back facet and $7.6 \%$ LR coating on the front facet, for increasing pump current. 
emitted from the front facet. Since the narrow and broad facets have similar transmission, this indicates that the photon density $(P)$ does not simply scale with the width $(w)$ of waveguide $(P . w \neq$ constant $)$. A rigorous modeling of the photon density inside the tapered laser is outside the scope of this paper. We thus rely on an experimental study to approximate the optimal front facet reflectivity. We obtain a maximum peak power of $6.2 \mathrm{~W}$ for the LR coating with $12 \%$ reflectivity. This corresponds to an increase of the power output by $67 \%$ compared to the uncoated device. The lowest reflectivity coating $(7.6 \%)$ has a reduced output power and is thus past the optimal value of reflectivity. We also note that for this last device the slope efficiency is reduced, which indicates that the laser experiences stronger waveguide losses (in addition to increased mirror losses). We attribute these losses to absorption in the $\mathrm{Al}_{2} \mathrm{O}_{3}$ quarter wave layer, since more intensity builds up inside the resonant layer.

A discontinuity in the slope efficiency can be observed in the L-I characteristics shown in Fig. 2(a) at high currents $(\mathrm{I} \approx 9.5 \mathrm{~A})$. This corresponds to the lasing threshold of a higher order lateral mode. We can observe from the LI curves that this threshold is affected by the coatings, although not in a straight-forward manner, as indicated by the absence of an observed higher order mode in the HR coated device measured. Scattering from facet defects and diffraction upon reflection are expected to influence the lateral mode structure since they are major coupling mechanisms between the different lateral modes. Both effects are modified by facet coatings. Previous works reported the use of anti-reflection coatings on the front facet of a tapered QCL in order to improve the beam shape. ${ }^{6}$

Figure 2(b) shows the far-field profiles of laser B with the final coating $(\mathrm{HR}+7.6 \% \mathrm{LR})$ for increasing drive currents. Apart from a small side lobe that grows with increasing drive current, the far-field is very stable. The $\mathrm{M}^{2}$ value is 1.6 at $\mathrm{I}=3.5 \mathrm{I}_{\mathrm{th}}$, where the maximum brightness of $4.05 \mathrm{MW} \mathrm{cm}^{-2} \mathrm{sr}^{-1}$ is reached. As discussed earlier, the side-lobe observed indicates the presence of higher order lateral modes.

Since the aperture of the laser at the end of the taper is very wide while its height remains bound by the active region thickness, the output beam has a very elongated crosssection, with a typical full-width at half-maximum of $6.1^{\circ}$ in the slow axis and more than $60^{\circ}$ in the fast axis. Furthermore, since the waveguide mode reaches the facet with a nonuniform phase profile along the lateral direction, it diverges in the slow axis from a virtual source that is approximately $L / n_{\mathrm{wg}}$ behind the output facet, where $L$ is the length of the taper and $n_{\mathrm{wg}}$ is the refractive index of the QCL waveguide. ${ }^{14}$ In contrast, the beam diverges from the output facet in the fast axis. This results in an astigmatism of the beam.

In an attempt to address these issues, we employed a strategy developed in our group to shape the output of edgeemitting QCLs into low-divergence beams using plasmonic collimators defined on their facets. ${ }^{15-17}$ Plasmonic collimators are composed of an aperture and a grating defined on the gold-coated facet of a semiconductor laser. The light coming from the laser waveguide passes through the aperture where it partially couples to surface plasmons (SP) propagating along the facet, which are in turn scattered by the grating grooves.
The grating can be viewed as an antenna array, with the fields scattered by the grooves and the field directly radiated into free-space by the aperture all constructively interfering to form a low-divergence beam. In addition to achieving lowdivergence output for standard edge emitting Fabry-Perot QCLs $\left(<4^{\circ}\right.$ in both vertical and horizontal directions $\left.{ }^{16}\right)$, plasmonic collimators can be used for polarization control ${ }^{18}$ and multi-beam emission. ${ }^{19}$ However, previous results on lowdivergence QCLs using plasmonic collimators showed substantial reduction of the power output (as much as $80 \%$ power reduction for the smallest divergence obtained ${ }^{16}$ ). This power reduction is caused by the low transmittivity of the aperture opened in front of the laser waveguide, especially for twodimensional collimators (collimating in both the fast and slow axis), which require a small aperture to diffract the SP waves and spread the energy laterally on the laser facet. ${ }^{16}$ Here, since the output beam already has a small lateral divergence because of the large width of the aperture $(100 \mu \mathrm{m})$, we only need a one dimensional collimator (with straight grooves and a broad aperture) in order to reduce the divergence along the fast axis. The large width of the aperture $(98 \mu \mathrm{m})$ results in a very small overlap between its lateral edges and the waveguide mode. Additionally, we chose the vertical dimension for the aperture $(4 \mu \mathrm{m})$ to be larger than the waveguide thickness $(3.5 \mu \mathrm{m})$ to reduce the overlap between the horizontal edges of the aperture and the laser waveguide mode. While this comes at the expense of a lower coupling to SP waves and results in a larger uncollimated background, we expect an increased power output compared to earlier collimator designs.

The fabrication method is similar to the one described in Ref. 16. Focus ion beam (FIB) milling was used to define 30 grooves into the $250 \mu$ m-thick InP substrate of a QCL (laser C). A 90-nm-thick $\mathrm{Al}_{2} \mathrm{O}_{3}$ film was then deposited for
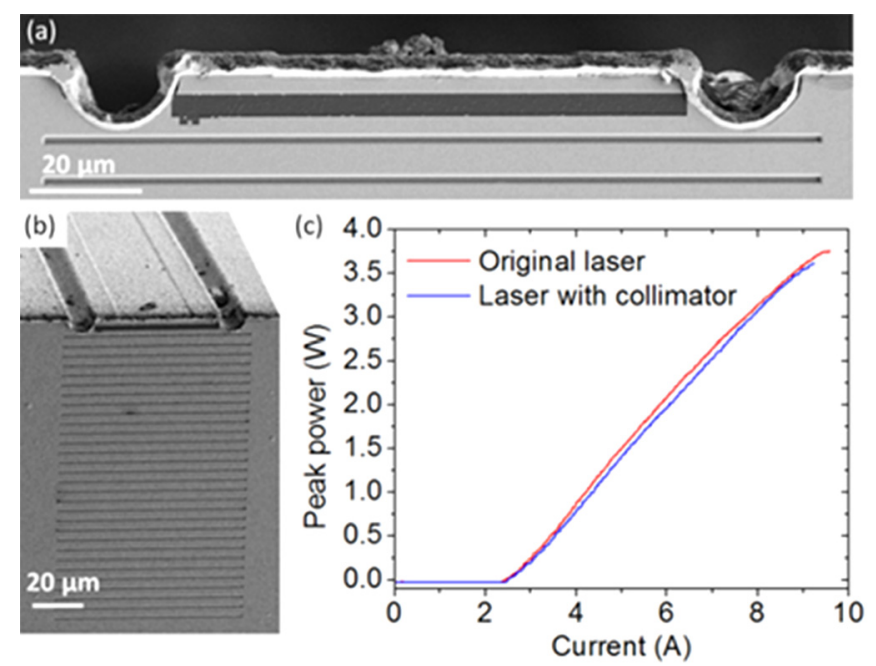

FIG. 3. (a) SEM image of a collimator fabricated on the facet of an uncoated tapered laser (laser $\mathrm{C}$ ), after deposition of $\mathrm{Al}_{2} \mathrm{O}_{3}$ and $\mathrm{Au}$ and subsequent opening of the aperture in front of the laser waveguide. (b) SEM image of the collimator. The substrate of the QCL $(250 \mu \mathrm{m})$ can accommodate 30 large grooves constituting a second-order grating with period $7.5 \mu \mathrm{m}$. The grooves are $130 \mu \mathrm{m}$ long, $1.7 \mu \mathrm{m}$ wide, and $1 \mu \mathrm{m}$ deep. After subsequent deposition of $\mathrm{Al}_{2} \mathrm{O}_{3}(90 \mathrm{~nm})$ and $\mathrm{Au}(250 \mathrm{~nm})$ by sputtering, the groove width is reduced to $1 \mu \mathrm{m}$. (c) LI curve of the device shown in (a) and (b), taken before and after fabrication of the plasmonic collimator. Both the collimated beam and a residual uncollimated background are measured by the powermeter placed directly in front of the laser. 

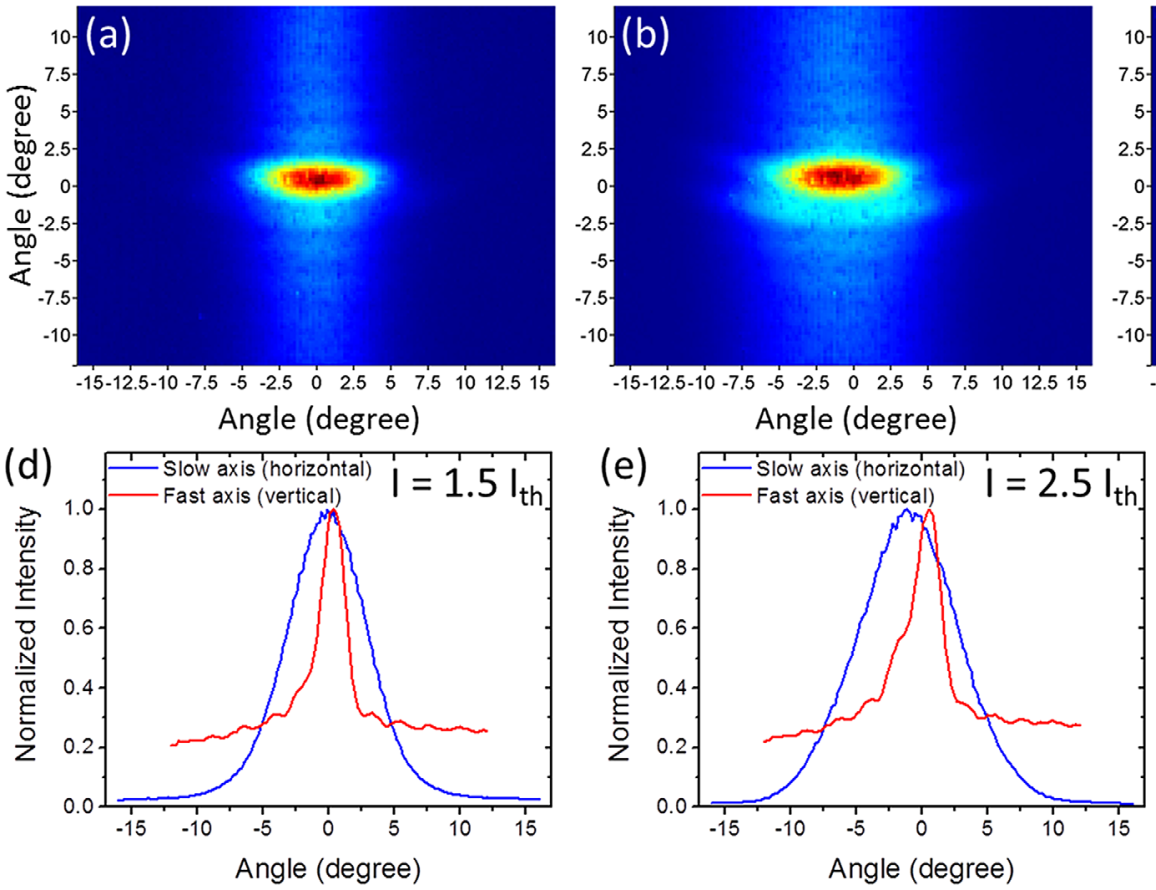
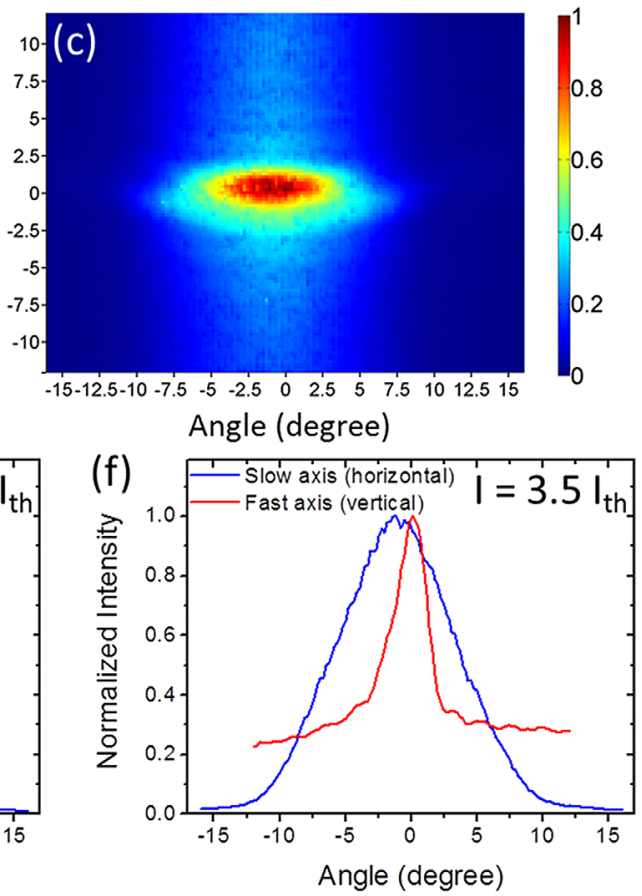

FIG. 4. (a)-(c) Two-dimensional far-field intensity distribution (normalized) of the tapered QCL with plasmonic collimator (laser C), measured using a midinfrared microbolometer array $(160 \times 120$ pixels, INO IRXCAM) with no optics placed directly in front of the laser facet. From (a) to (c), the pump current is, respectively, $1.5 I_{t h}, 2.5 I_{t h}$, and $3.5 I_{t h}$. The angles are computed from the known pixel pitch $(52 \mu \mathrm{m})$ and the distance between the facet and the sensor $(14 \mathrm{~mm})$. (d)-(f) Respective averages of the column and rows of the images shown in (a)-(c), showing the horizontal and vertical profiles of the beam.

electrical insulation, followed by a 250-nm-thick gold film. For both depositions, we used sputtering in order to obtain a quasi-conformal coating of the grooves. Finally, the aperture was opened using FIB. Fig. 3(a) shows a zoomed-in scanning electron microscope (SEM) image of the resulting aperture. An SEM image of the pattern facet is shown in Fig. 3(b). The grating design parameters are detailed in the caption. We observe in Fig. 3(c) the L-I characteristics of laser C, before and after fabrication of the collimator. The L-I characteristics are almost indistinguishable, demonstrating that the plasmonic collimator does not significantly affect the power output of the QCL. A maximum peak power of $3.6 \mathrm{~W}$ is reached for the collimated device. The maximum output power of previously reported plasmonic collimators is under $200 \mathrm{~mW}^{17}$

We measured the two-dimensional far-field profile of the collimated laser $\mathrm{C}$ for different driving currents, using a bare mid-infrared microbolometer array placed directly in front of the laser facet. The results are shown in Figs. 4(a)-4(c). We observe that the collimator results in a central low-divergence beam with FWHM of $7.1^{\circ}$ and $2.8^{\circ}$, respectively, along the slow and fast axis, for a driving current of $\mathrm{I}=1.5 \mathrm{I}_{\mathrm{th}}$. However, the directivity ${ }^{17}$ achieved is fairly low $(<15 \mathrm{~dB})$ because of the substantial uncollimated background whose level is between $20 \%$ and $30 \%$ of the peak intensity. As the current is increased, we observe a broadening of the central beam in the slow axis which could be caused by a narrowing of the intracavity lateral intensity profile.

In summary, we have demonstrated high-power, highbrightness QCLs based on $1^{\circ}$ tapered waveguides with sloped side-walls. Their output power was optimized using a combination of HR and LR coatings, reaching a maximum peak power of $6.2 \mathrm{~W}$. Plasmonic collimators were employed on the original uncoated tapered lasers to reduce the large fast axis beam divergence and obtain a more circular beam shape, which may circumvent the need for external beamshaping optics in applications such as high-efficiency coupling into mid-infrared optical fibers. The high power output was preserved in the presence of the plasmonic collimators.

We acknowledge the support from DTRA (Contract No. HDTRA1-10-1-0031) and partial financial support from Hamamatsu Photonics KK. This work was performed in part at the Center for Nanoscale Systems (CNS) at Harvard University, a member of NNIN, which is supported by the NSF. M.K. and T.M. are supported by the NSF GRFP.

${ }^{1}$ R. F. Curl, F. Capasso, C. Gmachl, A. A. Kosterev, B. McManus, R. Lewicki, M. Pusharsky, G. Wysocki, and F. K. Tittel, Chem. Phys. Lett. 487, 1 (2010).

${ }^{2}$ R. Martini, C. Bethea, F. Capasso, C. Gmachl, R. Paiella, E. A. Whittaker, H. Y. Hwang, D. L. Sivco, J. N. Baillargeon, and A. Y. Cho, Electron. Lett. 38, 181 (2002).

${ }^{3}$ A. Lyakh, R. Maulini, A. Tsekoun, R. Go, S. Von der Porten, C. Pflügl, L. Diehl, F. Capasso, and K. N. Patel, Proc. Natl. Acad. Sci. U.S.A. 107, 18799 (2010).

${ }^{4}$ Y. Bai, N. Bandyopadhyay, S. Tsao, S. Slivken, and M. Razeghi, Appl. Phys. Lett. 98, 181102 (2011).

${ }^{5}$ B. Gokden, T. S. Mansuripur, R. Blanchard, C. Wang, A. Goyal, A. Sanchez-Rubio, G. Turner, and F. Capasso, Appl. Phys. Lett. 102, 053503 (2013).

${ }^{6}$ L. Nahle, J. Semmel, W. Kaiser, S. Hofling, and A. Forchel, Appl. Phys. Lett. 91, 181122 (2007).

${ }^{7}$ Y. Bai, S. Slivken, Q. Y. Lu, N. Bandyopadhyay, and M. Razeghi, Appl. Phys. Lett. 101, 081106 (2012). 
${ }^{8}$ K. Fujita, S. Furuta, A. Sugiyama, T. Ochiai, T. Edamura, N. Akikusa, M. Yamanishi, and H. Kan, IEEE J. Quantum Electron. 46, 683-688 (2010).

${ }^{9}$ G. Hatakoshi, Opt. Rev. 10, 307 (2003).

${ }^{10}$ D. Bisping, D. Pucicki, M. Fischer, J. Koeth, C. Zimmermann, P. Weinmann, S. Hofling, M. Kamp, and A. Forchel, IEEE J. Sel. Top. Quantum Electron. 15, 968 (2009).

${ }^{11}$ R. Maulini, A. Lyakh, A. Tsekoun, R. Go, C. Pflügl, L. Diehl, F. Capasso, and K. Patel, Appl. Phys. Lett. 95, 151112 (2009).

${ }^{12}$ Y. Bai, S. R. Darvish, N. Bandyopadhyay, S. Slivken, and M. Razeghi, J. Appl. Phys. 109, 053103 (2011).

${ }^{13}$ E. D. Palik, Handbook of Optical Constants of Solids (Academic Press, New York, 1991).
${ }^{14}$ J. N. Walpole, Opt. Quantum Electron. 28, 623 (1996).

${ }^{15}$ N. Yu, J. Fan, Q. Wang, C. Pflügl, L. Diehl, T. Edamura, M. Yamanishi, H. Kan, and F. Capasso, Nat. Photonics 2, 564 (2008).

${ }^{16}$ N. Yu, R. Blanchard, J. Fan, F. Capasso, T. Edamura, M. Yamanishi, and H. Kan, Appl. Phys. Lett. 93, 181101 (2008).

${ }^{17}$ N. Yu, R. Blanchard, J. Fan, Q. Wang, C. Pflügl, L. Diehl, T. Edamura, S. Furuta, M. Yamanishi, H. Kan, and F. Capasso, IEEE Trans. Nanotechnol. 9, 11 (2010)

${ }^{18}$ N. Yu, Q. Wang, C. Pflügl, L. Diehl, F. Capasso, T. Edamura, S. Furuta, M. Yamanishi, and H. Kan, Appl. Phys. Lett. 94, 151101 (2009).

${ }^{19}$ J.-P. Tetienne, R. Blanchard, N. Yu, P. Genevet, M. A. Kats, J. A. Fan, T. Edamura, S. Furuta, M. Yamanishi, and F. Capasso, New J. Phys. 13, 53057 (2011). 